\title{
Assessment of tunnel perimeters produced by blasting
}

\author{
A.T. Spathis Orica Mining Services Technical Centre, Australia \\ G. Yuill Orica Mining Services Technical Centre, Australia \\ D. Stow Orica Mining Services Technical Centre, Australia
}

\begin{abstract}
Over break and under break are important measures of conformance to the designed perimeter in a civil or mine tunnel excavated by blasting. Three randomised paired trials of a new low-density bulk explosive compared with $70 \mathrm{~g} / \mathrm{m}$ detonating cord loaded in the perimeter were made in different development headings of an underground zinc mine. The low-density bulk perimeter explosive produced more over break but less under break in two of the three comparisons with the detonating cord. However, a statistical analysis on the small number of paired comparisons indicated insufficient power in a paired t-test and further paired comparisons are recommended. Rather than the few well defined half-barrels of the detonating cord, characteristic scorch/crush zones were evident in the low-density bulk explosive rounds and miners observed somewhat increased barring down time in these blasts. Such observations are understandable due to the higher effective perimeter charge of the low-density bulk perimeter explosive. The maximum measured over break was less than 12\% and the maximum measured under break was less than $8 \%$ for either blasting system. The miners stated that the speed of loading full faces was faster with the low-density bulk explosive system, compensating for longer barring-down times. Work is ongoing to improve the low-density bulk explosive as an alternative for mines seeking rapid development mining and tunnelling.
\end{abstract}

\section{Introduction}

The measurement of the effectiveness of a tunnel excavation in mining often relies on direct visual inspection of the post-blast perimeter. The visual assessment occurs prior to and during the surface barring down and the introduction of ground support. Some of these processes may be mechanical or manual. The visual assessment is usually in terms of the presence or absence of half-barrels. This is sometimes quantified in a half-barrel factor (HBF) that represents the percentage of observed half-barrels out of the total length expected from the number of perimeter blastholes drilled. It is recognised that the HBF does not assess the damage condition of the rock mass adjacent to the excavation (Paventi et al., 1996).

An alternative and perhaps complementary approach to the assessment of tunnel perimeters is to survey the final excavation and compare it to either the as-designed perimeter or to a constructed as-drilled perimeter (Yamamoto et al., 1995; Spathis et al., 2006; Wetherelt and Williams, 2006). Ideally, this is best done in three dimensions so that the volume of over break or under break may be characterised. Modern scanning laser instruments make the data collection fast and reliable.

Such survey methods are applied to the assessment of different explosives, initiation systems and blast designs and use the information to determine the effectiveness in producing tunnel perimeters that conform to the as-designed perimeter or to the as-drilled perimeter. The present paper reports a case study where a comparison was made between an existing blast design with detonating cord in the perimeter versus a new low-density bulk perimeter product. Randomised paired comparisons are used to assist the assessment. The motivation for the introduction of the new product is that the detonating cord:

- May not be consumed totally in a blast.

- May pose a security risk as it is relatively portable.

- Is relatively slow to load and it has a relatively high cost. 


\section{$2 \quad$ Blasting trial methodology}

The work presented here is part of an ongoing research and development program to design blasting systems that deliver improved extraction of tunnel rounds in civil and mine site projects (Stow et al., 2008). It is accepted widely that reducing the energy in the blastholes at or close to the perimeter of a planned excavation produces better results (Persson et al., 1994). Common practices are to use decoupled charges or lower density explosives in such blastholes, sometimes coupled with precise timing provided by electronic delay detonators (Spathis et al., 2006). The present trials were conducted with a new bulk perimeter explosive that offers the benefits of effective low-density and also fast loading into the blastholes using bulk delivery systems.

The experimental process used to determine any difference between one blasting method and another was the application of randomised paired comparisons (Box et al., 1978). This approach has been adopted in blasting trials at mine sites (Noy and Brent, 2003; Brent and Noy, 2005). In essence this attempts to control variables that are within the gift of the engineer and to block against such factors while randomising across factors that may vary but over which there is little or no control. In blasting this is often the geology, drilling crew or charging crew, for example. The intent is to use adjacent blasts, or where possible a single blast, where the reference and modified blasting method are used. In mine development, and for these trials, adjacent blasts of detonating cord and of low-density bulk explosive in the perimeter blastholes were used for the paired comparisons. It is desirable to use the reference and modified blasting method in a single blasting round as performed by Yamomoto et al. (1995). However, in a working civil tunnel or mine project, the logistics make it difficult to use such mixed explosive loading. Also, the cut region is usually not centralized for safety reasons and this may have undue influence on the paired comparisons. The present trials adopted the use of adjacent blasts as the next best alternative.

Blast performance assessment involved two separate techniques: the first was a visual approach whereby an experienced mine person and an experienced Orica person familiar with the mine each categorised their view of the acceptability of the drilling and the blast result; the second approach was based on detailed survey data whereby a comparison is made between the expected perfect as-drilled volume and the as-blasted volume. The drilling, charging and scaling crews also provided feedback during the trials. Those comments were unstructured but related to the quality of drilling, ease of charging and the speed of scaling after a blast.

The visual impressions were documented essentially as either acceptable or not acceptable. Half-barrels were noted if present. Three-dimensional surveys of the perimeter blastholes and of the post-blast cavity were conducted. The visual review gave a qualitative assessment while the survey data provided a quantitative assessment. The latter approach enables measured data to be interpreted in the form of percentage volume over break and percentage volume under break. The two as-drilled and as-blasted objects can be overlaid on each other and this provides an alternative visual assessment.

\subsection{Pre-blast survey}

The pre-blast survey was undertaken in order to assess drilling quality and accuracy as well as to provide the information to construct an as-drilled object for the modelling and analysis stages of the evaluation process.

The process steps comprised:

- Fixing the location of a total station to mine coordinates by resection with known survey stations.

- Placing known length rigid rods in each perimeter blast hole (Figure 1).

- Surveying the location of hole collars and the ends of each rod using a total station.

- Removing rods from holes using a scissor lift or basket-equipped vehicle.

\subsection{Post-blast survey}

The post-blast survey was undertaken to record blast performance data and provide an as-blasted object for the modelling and analysis stage of the evaluation process. 
The process steps comprised:

- Fixing the location of a total station to mine coordinates by resection with known survey stations.

- Setting up a 3D scanner and reference prism as per safe working practice.

- Surveying the location of the scanner and reference prism.

- Running the scanner to obtain a 3D model of the cavity formed by blasting.

\subsection{Blast performance analysis process}

The analysis proceeded in four simple phases:

- Firstly, the surveyed collar positions and those of the end of a fixed length rod placed in each perimeter blasthole were used to project to the three dimensional toe positions. Thus, the surveyed collar positions and the deduced toe positions were used to construct the blasthole in three dimensional spaces, assuming the perimeter blastholes are straight. These blastholes were used to construct a three dimensional as-drilled object.

- Secondly, the post-blast cavity was surveyed using a Callidus 3200 laser scanner and its data was also placed in mine coordinates. This represented the as-blasted object.

- Thirdly, the two surveys were overlaid and the Boolean union was formed from the two objects. Essentially this was the space occupied by both the as-drilled and as-blasted objects.

- Finally, a difference between the Boolean union and each of the as-drilled and as-blasted objects yielded the over break volume and the under break volume.

Over break refers to that volume of the as-blasted cavity that lies outside the as-drilled object. Under break refers to that volume of the as-blasted cavity that lies inside the as-drilled cavity. In order to reduce the end-effects and also floor conditions, the two objects were truncated by two approximately vertical planes whose normal lies in the direction of the tunnel and by one approximately horizontal plane that was slightly above the actual floor.

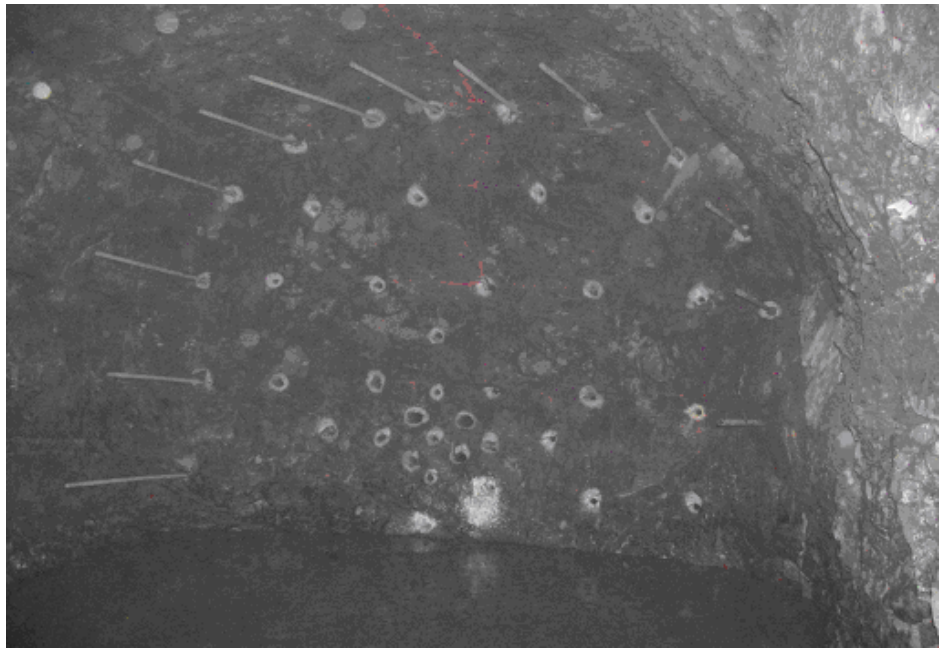

\section{Figure 1 Poles placed in perimeter holes ready for survey}

\section{Blast performance results}

The blasting was conducted in mine development for an orebody that lies in lower carboniferous limestones. The minerals types are sphalerite (zinc-bearing) and galena (lead-bearing). An increase in mine production has demanded significant mine development and improved excavation equipment and methods. Currently, the primary mining method is open-stoping with draw points in the footwall. Remote control technology is used wherever possible. 
The logistics of conducting the blast performance trials were challenging:

- The blasting rounds assessed were in different parts of the mine and fired on different shifts.

- Some rounds had a pre-blast survey but not a post-blast survey.

- Some rounds did not allow adjacent rounds to be fired with the two alternative products.

Visual assessments of ten low-density bulk product blasts were made. These included comments on the drilling accuracy, the visibility of any half-barrels, the extent of scaling required and an overall comment on the round acceptability. Of these ten blasts, just three had a matching adjacent reference blast so that three paired comparisons could be made. One further low-density bulk product blast had a full set of survey data for evaluation, but without a matching pair of the detonating cord perimeter blasting system.

The visual assessment of blast results by the mine was quite different to that of Orica. The mine person assigned to assess overall product performance found only one of the low-density bulk explosive shots to be acceptable. Nine of the low-density bulk explosive shots were deemed unacceptable. Orica personnel regarded seven of the blasts to be acceptable (including the one deemed acceptable to the mine person) and three unacceptable. A hole density well above the target range of $0.4-0.6 \mathrm{~g} / \mathrm{cc}$ was recorded for one of the unacceptable blasts. Advance on another was only half that expected, an outcome not related to the use of the low-density bulk explosive. One blast was loaded into poor ground, where detonating cord may have produced unsatisfactory results. These last three blasts were not involved in the paired comparisons.

A visual assessment of the presence of half-barrels was also made. These were categorised with statements by the observers like sign of half-barrels and none. Some half-barrels were observed in almost all blasts but the low-density blasts produced characteristic scorch/crush zones typical of higher energy in the vicinity of the perimeter blastholes. The scaling crews found the blasts that used the higher energy product required more scaling. The charging crew suggested that the loading of the low-density bulk product was faster and simpler than when loading perimeter blastholes with detonating cord.

The quantitative results from survey data produced a different assessment and these are described below. Also, the drilling data is usually compared to as-designed blast patterns. These were not available so the data has been analysed by looking at the distribution of blasthole lengths, collar spacings and toe spacings. Figure 2 shows box and whisker plots for the single case of poor drilling. The centre vertical line is the median value; the box defines where $50 \%$ of the values about the median lie and the other vertical bars define the extreme values. Figure 2(a) shows that the median length of the blastholes is approximately $4.5 \mathrm{~m}$ and that most blastholes are acceptable. The collar spacings are clustered around $0.8 \mathrm{~m}$ and the toe spacings are around $1.1 \mathrm{~m}$. Clearly, in this case some toe spacings are unacceptably large and it would be expected that this blasting round would produce poor results when compared to the planned excavation design. However, the data indicates quite good drilling control for all other blasts.

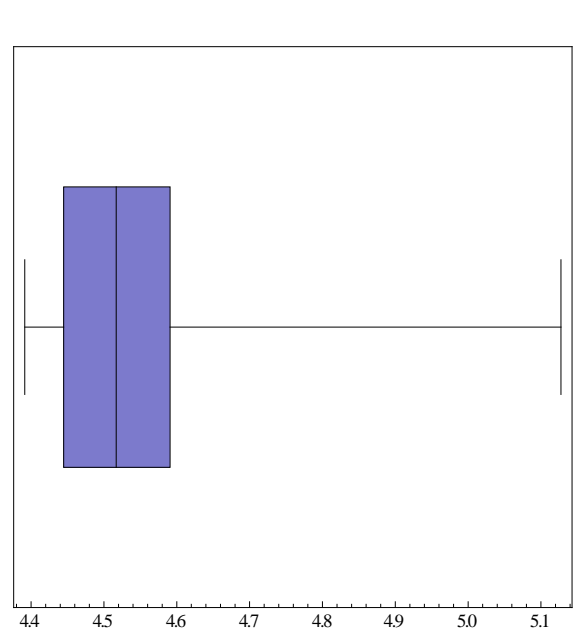

(a)

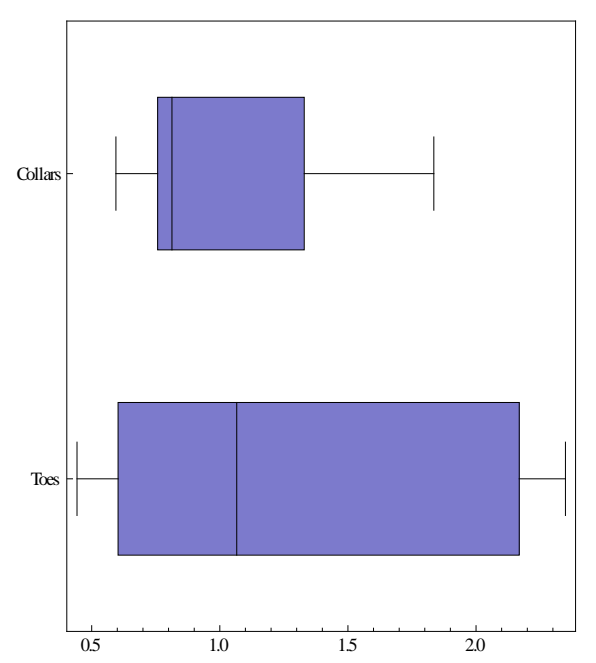

(b)

Figure 2 Box and whisker plots: (a) hole lengths (m); (b) collar and toe spacings (m) 
Six rounds were surveyed as part of a paired comparison. That is, surveys were conducted of a pair of shots in the same heading, one directly after the other consisting of a control round of the mines current practice of detonating cord in the perimeter and an experimental round of the low-density bulk explosive system in the perimeter blastholes. The data from the paired comparisons are shown below plus one other result from a single low-density bulk explosive shot where complete survey data was available.

The blasthole traces and the formed as-drilled object are shown in Figure 3(a) for a typical round. The overlay of the as-blasted cavity, as-drilled object and the blasthole traces are shown in Figure 3(b). In both, the information is shown after the near-vertical and near-horizontal truncations that are used to minimise end effects and poor mucking of the floor. The union object is merely the space occupied by either the as-drilled object, the as-blasted object or space occupied by them both. Differences between the union and the other two are used to form the over break and under break data.

The over break and under break percentage volumes are shown in Figures 4(a) and 4(b), respectively. The lack of data for the detonating cord case of the fourth blast pair is unfortunate as it reduces the pairs available for comparison of the two methods. For the cases where paired comparisons were available it can be seen that the over break percentage is greater in two of the three cases for the modified blasting method (lowdensity bulk) compared to the reference blasting method. The under break results are shown in Figure 4(b). Here, in two of the three cases where pairs exist, the modified blasting method (low-density bulk) had less under break than did the reference blasting method.

The isolated result from blast four is worth examining. It yielded the largest over break and the largest under break of any of the data in the matched pairs. However, given that it has no pair fired in the same (adjacent) ground using a reference blast configuration, no further information can be drawn regarding how these relatively higher over break and under break relate to the other data from the paired comparisons. This highlights the need to use a structured approach to blast performance assessment — particularly, as it is difficult to obtain data in a producing mine or civil tunnel project.

As might be expected, given the variability in the over break and under break data from one blast to the next, and also that just three paired comparisons exist, no significant difference is indicated in a paired t-test on the paired data. The paired t-test analysis, including the statistical power of the data, is in Table 1 (R Development Core Team, 2008):

- " $n$ " is the number of paired comparisons.

- "delta" is the mean of the paired differences.

- "sd" is the standard deviation of the paired differences.

- "sig.level" is the significance level that is typically chosen to be 0.1 or 0.05 .

- "confidence interval" is the range in which it is expected the true mean difference to lie given the significance level.

- "p-value" is the probability for testing the null hypothesis and for fixed significance level, the null hypothesis is rejected when the p-value is less than the significance level.

- "power" is the probability of avoiding rejection of the null hypothesis when it is false. Increasing the number of paired comparisons used in the analysis may increase the "power".

- "alternative" indicates the alternative to the null hypothesis: the treatment may produce an increase or decrease in over break or under break and either or both possibilities are allowed in the t-test.

In the present case, a two-sided alternative is appropriate for the null hypothesis and mean differences may be greater or less than zero. 
Table 1 Paired t-test calculations for over break and under break data

\begin{tabular}{ll}
\hline Over break & Under break \\
\hline $\mathrm{n}=3$ & $\mathrm{n}=3$ \\
delta $=1.73$ & delta $=-0.067$ \\
$\mathrm{sd}=3.65$ & $\mathrm{sd}=1.38$ \\
sig.level $=0.1$ & sig.level $=0.1$ \\
confidence interval = 1.43 to 2.03 & confidence interval $=-0.18$ to 0.047 \\
p-value $=0.50$ & p-value $=0.94$ \\
power $=0.14$ & power $=0.06$ \\
alternative $=$ two-sided & alternative $=$ two-sided \\
\hline
\end{tabular}

A "sig.level" of 0.1 was chosen, which means that should the null hypothesis of equal means for the reference and modified blasting method be rejected, there is a probability of $10 \%$ that the results of the data are due to chance alone. As can be seen, the "power" is about $14 \%$ for the over break data and $6 \%$ for the under break data. These are well below the value of $80 \%$ commonly used (Park, 2008). At this "sig.level” the "power" is weak and this means that more paired tests are required to obtain a definitive statistical analysis. The "p-value" of 0.50 for the over break ( 0.94 for the under break) data is much larger than 0.1 and the hypothesis that the mean value of the differences in the paired data equals zero cannot be rejected. This does not imply that the mean value of the over break (or under break) produced by the low-density bulk product is equal to the mean value of the over break (or under break) produced by the detonating cord blasts. There still may be a difference but the experiments did not have the capacity to expose it. Indeed, in previous related work it was suggested that such differences were produced by the more energetic explosive system (Stow et al., 2006). That work used a linear and areal analysis rather than the volumetric analysis here. It also used a different laser scanning protocol.

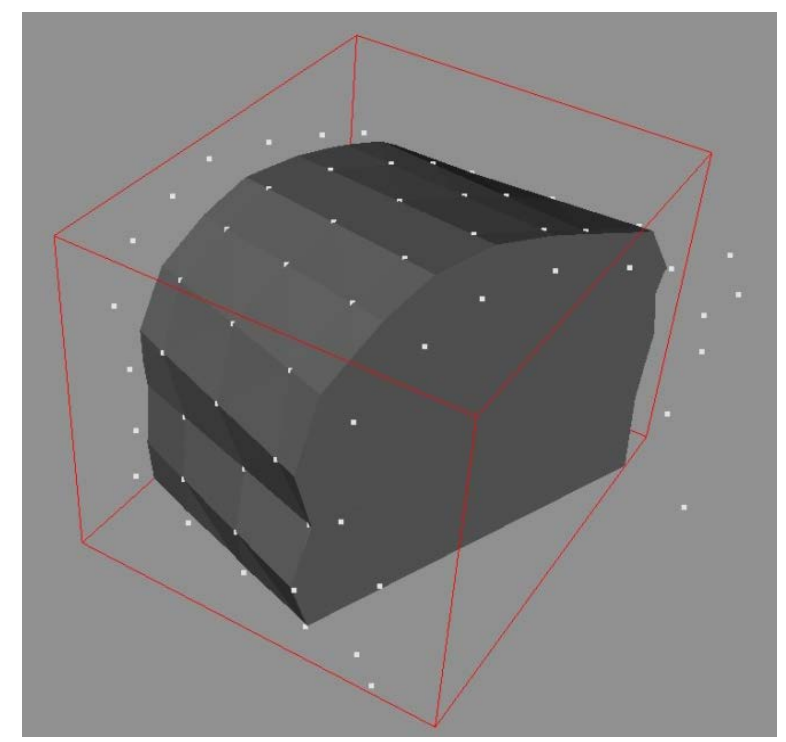

Figure 3 (a) Overlay of the perimeter blasthole traces (white dots) and the associated as-drilled object formed by connecting the blastholes. The blasthole traces are the edges of the object. The object has been truncated at the front and rear of the blastholes and also at the floor 


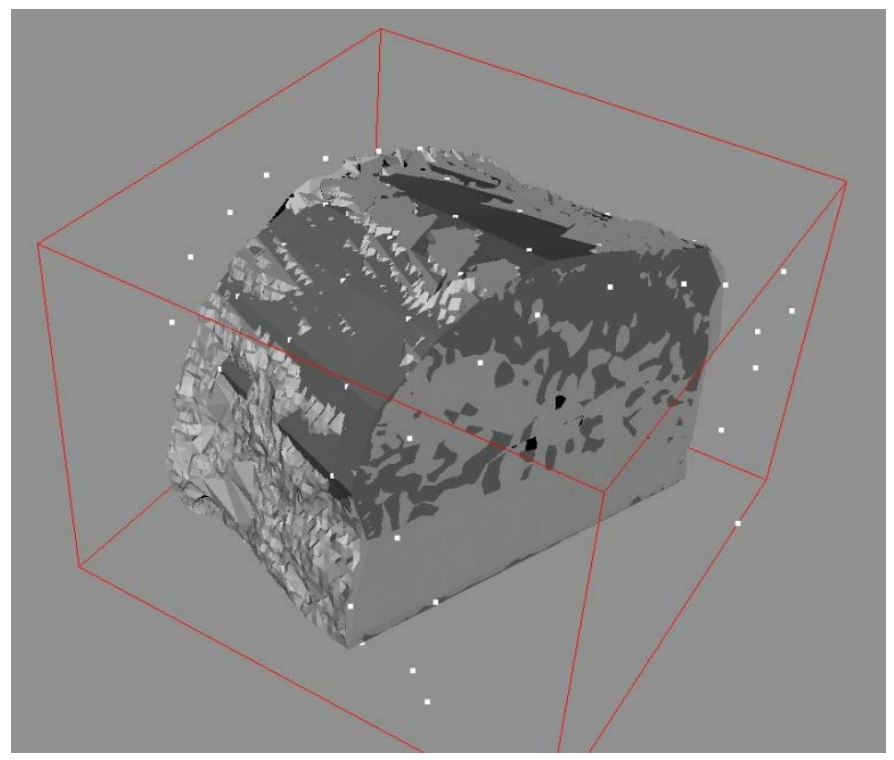

Figure 3 (b) Overlay of the as-blasted cavity, the blasthole traces and the as-drilled object. The two objects have been truncated. From this view it can be seen that the as-blasted cavity lies outside the as-drilled object and hence represents a level of over break, with some under break shown in this view

Over break Volume

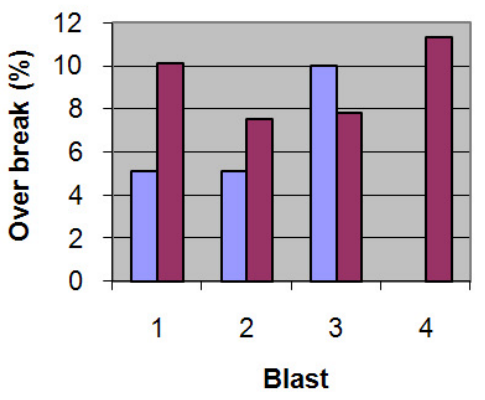

(a)
Under break Volume

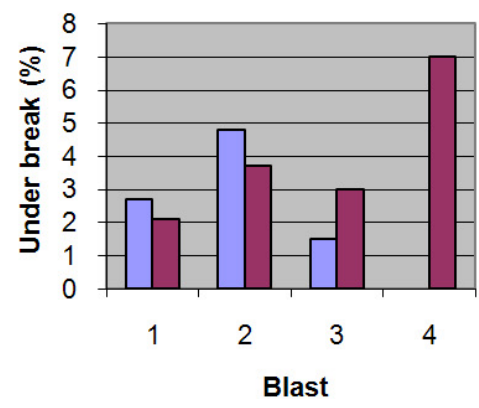

(b)

Figure 4 (a) Over break percentage obtained from a number of blasts; (b) under break percentage obtained from a number of blasts

\section{Conclusions}

The statistical analysis shows that more paired comparisons are required in order to avoid accepting that there is no difference in the two blasting methods in terms of over break and under break, when indeed there may be. It is worth noting that in previous work at the same mine, but using different geometric analysis and measurement procedures, there was some evidence that there were differences in the two blasting methods. Unfortunately, the differences in the geometric analysis and measurement procedure preclude combining the data of both studies. In the earlier work, the low-density bulk product in perimeter blastholes produced greater over break and less under break than did the detonating cord used in perimeter blastholes (Stow et al., 2006). Note that in both sets of studies, no other modifications, apart from the perimeter product, were made in the blast design so that while the blast design was optimised for the standard practice of detonating cord in perimeter blastholes, it was not necessarily the case for the low-density bulk product. It is important to note the quite large differences in the charge mass per metre of blasthole occur for each of the perimeter products. The detonating cord was $70 \mathrm{~g} / \mathrm{m}$ while the nominal low-density bulk explosive product was around $900 \mathrm{~g} / \mathrm{m}$. 
Drilling is a significant contributor to the blast result and in the data the majority of the drilling was in good control. No blast design data was available at the time of writing and this is not unusual in an operating mine where the rate of tunnel development is high and the blast is designed the same as the previous round. In any case, comparisons are always best done between the as-drilled information and the as-blasted survey data. This helps to include the influence of the drilling accuracy into the blast performance assessment - one cannot expect blasting to correct for poor drilling.

Visual observations are used routinely and involve aspects of local experience that cannot be reproduced in a raw geometric survey. However, perceptions may vary between observers, and it is important to move to methods that are quantitative, provided these deliver the information required by the mine or tunnel owner. It is suggested that one such approach is to use the type of survey methods and analysis described here.

Despite evidence for increased barring down times for blasts using the low-density bulk explosive system, it was evident that loading times were faster using the new bulk system than with detonating cord faces. Part of the increased scaling times is almost certainly due to the extra energy in the low-density bulk system. The use of randomised paired comparisons in mining trials is advocated wherever possible as is the application of the survey processes used in this paper. The data shows that neither system produced an over break greater than $12 \%$ or an under break greater than $8 \%$. Work is ongoing to improve the low-density bulk explosive system as an alternative for mines seeking rapid development mining and tunnelling.

\section{Acknowledgements}

The authors would like to thank the mine and the Orica people involved in the blasting trials. The mine provided not only logistical support but modified their normal practices to enable the trials reported here. The authors thank their colleagues, Les Armstrong, Geoff Brent and Mike Noy, for their review of the paper. The authors also thank Geoff and Mike for their comments on the statistical analysis.

\section{References}

Box, G.E.P., Hunter, W.G. and Hunter, J.S. (1978) Statistics for Experimenters, John Wiley and Sons, New York, pp. 93-106.

Brent, G.F. and Noy, M.J. (2005) Matched pairs blasting technique to quantify the benefits of the i-kon ${ }^{\mathrm{TM}}$ electronic $^{\mathrm{s}}$ initiation system, Proceedings of the Third EFEE World Conference on Explosives and Blasting, R. Holmberg (ed), Brighton, September 13-16, pp. 481-492.

Noy, M.J. and Brent, G.F. (2003) Mine site trialling — experimental design and measurement methods, Orica Technical Report No. 58635.

Park, H.M. (2008) Hypothesis Testing and Statistical Power of a Test. Technical Working Paper, The University Information Technology Services (UITS), Center for Statistical and Mathematical Computing, Indiana University.

Paventi, M., Lizotte, Y., Scoble, M. and Mohanty, B. (1996) Measuring rock mass damage in drifting, Proceedings of the Fifth International Symposium on Rock Fragmentation by Blasting, B. Mohanty (ed) August 25-29, Montreal, Canada, A.A. Balkema, Rotterdam, pp. 131-138.

Persson, P-A., Holmberg, R. and Lee, J. (1994) Rock Blasting and Explosives Engineering, CRC Press Inc., Boca Raton, pp. 265-272.

R Development Core Team (2008) R: A language and environment for statistical computing, R Foundation for Statistical Computing, Vienna, viewed on 20 February 2009, http://www.R-project.org.

Spathis, A.T., Armstrong, L.W. and Lesberg, P.L. (2006) Tunnel blasting using precise electronic delay detonators and bulk emulsions, Proceedings of the Eighth International Symposium on Rock Fragmentation by Blasting, May 7-11, Santiago, Chile, pp. 411-416.

Stow, D., Yuill, G. and Spathis, A.T. (2008) A Second Implementation of Bulk Perimeter Product, Orica Internal Technical Report, March.

Stow, D., Yuill, G. and Spathis, A.T. (2006) Blasting Trials of Bulk Perimeter Product, Orica Internal Technical Report No. 58763.

Wetherelt, A. and Williams, D.C. (2006) Using high definition surveying (HDS) to quantify tunnel hole burdens and fragmentation, Proceedings Eighth International Symposium on Rock Fragmentation by Blasting, May 7-11, Santiago, Chile, pp. 55-60.

Yamamoto, M., Ichijo, T. and Tanaka, Y. (1995) Smooth blasting with electronic delay detonator, Proceedings Eleventh Annual Symposium on Explosives and Blasting Research, International Society of Explosives engineers, February 5-9, Nashville, Tennessee, pp. 144-156. 